$\xi=-1$

\title{
End-to-End Performance Evaluation for MIMO-OSTBC Adaptive Modulation Over Rayleigh Fading Channel
}

\author{
Alauddin Al-Omary \\ Department of Computer Engineering, College of IT, University of Bahrain \\ *Corresponding author E-mail: aalomary@uob.edu.bh
}

\begin{abstract}
This paper presents end-to-end performance evaluation for adaptive Orthogonal Space-Time Block Codes (OSTBC) Multiple Input Multiple Output (MIMO) communication system over frequency selective Rayleigh fading channel. The system operates with one, two, three or four transmit or receive antennas to transmit and receive any frame. The system uses an adaptation algorithm that dynamically adjusts the used number of transmit and receive antennas based on the difference between the target and actual frame-error rates (FER) of the overall system. In this way, the performance of the system can be controlled adaptively. Alamouti and other adaptation algorithms are implemented in this system. The results show that FER improve significantly as the SNR increases and when the SNR is small, the adaptive algorithms successfully adjust the use of MIMO antenna to compensate the impairment caused by the faded and noisy channel.
\end{abstract}

Keywords: Adaptive Modulation, MIMO, OSTBC, FER, OFDMA

\section{Introduction}

5G networks require high-speed low latency wireless diverse services. The diverse service offers "anywhere anytime connection" and requires efficient, robust and spectrally efficient wireless data transmission. Wireless channels suffer from channel deficiencies such as fading and interference, which distort the received signal [3]. Natural obstacles such as trees or artificial obstacles such as buildings may get in the way of the radio frequency signal. Thus, the signal may suffer from reflection, refraction, diffraction or superposition, and this may cause inter-symbol interference (ISI) distortion that results from the arrival of multi copies of the transmitted signal at the receiver at different times. ISI occurs if the delay spread is greater than the symbol period of the modulation scheme [7-11]. Several frequency selective fading channel impairments can be reduced using a combination of diversity of various forms but cannot solve the problem of ISI [4]. Chen-nee et al [5] used coding techniques to preserve satisfactory performance in deep fades but these techniques are not effective in solving the ISI problem [5]. Another method that can be used to solve ISI and fading problems is using single user MIMO that uses multiple antennas at the transmitter and receiver to introduce spatial diversity that increases the capacity and reduces the bit error rates [3], [12] and [13]. This technique is helpful but it did not utilize the channel state information (CSI) to eliminate the effect of fading [6]. Multi-user MIMO (MU-MIMO) is an enhancement of MIMO systems but it requires sufficiently accurate CSI at the transmitter to realize spectral efficient transmission. One well-known problem of the MIMO systems is that the feedback channel affects the CSI quality. As the CSI distortion increases, the performance of the MIMO systems degraded because the quality of the MU-MIMO precoder that controls the inter-user interference is reduced [14].

Space-Time Block Codes (STBCs) [1], [15] are appealing techniques for MIMO wireless communications. They exploit full spatial diversity order and they have simple decoding that take advantage of symbol-wise maximum likelihood (ML) technique. This paper presents end-to-end performance evaluation for adaptive Orthogonal Space-Time Block Codes (OSTBC) MIMO over frequency selective Rayleigh fading channel. The number of transmit and receive antennas of the proposed system are adaptively adjusted based on the difference between a target and the actual FER of the system. Alamouti and other adaptation algorithms are implemented in this system [1], [2]. The results show that FER improve significantly as the SNR increases and when the SNR is small, the adaptive algorithms successfully adjust the use of MIMO antenna to compensate the impairment caused by the faded and noisy channel. By adapting the use of MIMO antenna in transmitter and receiver, the system performance can be adjusted and FER can be controlled because MIMO utilizes rich scattering environment that increases the channel capacity. In this paper, section two presents the literature review. The scope of the work is shown in section three. Section four presents the channel and OSTBC models. Section five presents the system overview and finally section six presents the simulation setup and results.

\section{Literature Review}

MIMO systems represent one of the most important technical breakthroughs in modem communication systems. MIMO is a system that uses multiple transmitter antennas and multiple receiver antennas. Theoretically, MIMO systems can provide very high capacity that may grow approximately linear with the number of antennas. The main concept of the MIMO systems is its ability to change multi-path propagation, which is normally a problem in conventional wireless communications, into an advantage for users. In MIMO systems, the channel capacity and the channel reliability can be enhanced. By using spatial multiplexing, the capacity of the channel can be enhanced whereas the channel reliability may be enhanced by using space time coding (STC). STBC was analyzed first by Tarokh [19] and then was modified by Alamouti [1] who introduced the maximum likelihood (ML) 
technique to simplify the receiver design. MIMO has many advantages such as Beamforming, spatial diversity and spatial multiplexing [24], [25]. STBC can improve performance through measuring the average number of channels fully utilized by each piece of information transmitted, which is known as diversity. The maximum diversity available to a space-time system is $N t$. $N r$, which is the total number of channels between $N t$ transmitters and $N r$ receivers. Having many channels between transmitter and receiver can be viewed as redundancy in case other channels crashed. Researchers proposed many schemes for the transmission of signals via MIMO systems. Among these schemes are the Block Layered Space-Time (BLAST) [18], Space-Time Trellis Codes (STTCs) [19], Space-Time Block Codes (STBCs) by Alamouti in 1998 [1], [2] and Unitary Space-Time Codes [19] among many others. These schemes aimed to achieve diversity and highly possible data rates while keeping bandwidth expansion as small as possible.

Orthogonal STBCs are currently receiving an intensive attention due to the following reasons:

1. Due to their orthogonally, they simplify the decoding at the receiver side.

2. A good error probability characteristic can be achieved if a full diversity order for a certain number of $\mathrm{Tx}$ antennas are provided [2].

Alamouti proposed a code for MIMO with two antenna [1]. Tarokh et al. [2] extended Alamouti work by proposing STBCs for more than two Transmitter antennas. Tarokh classified STBCs into two classes namely "STBCs for real signals" and "STBCs for complex signals". Real STBCs can be used with Pulse Amplitude Modulation (PAM) while complex STBCs are used for Phase Shift Keying (PSK) or Quadrature Amplitude Modulation (QAM) constellations. A complex STBC is also known as an orthogonal STBC (OSTBC).

Real STBCs have been examined intensively by researches. Researches set a systematic method to construct real STBCs with the maximum rate from 1 to 8 Transmitter antennas based on HuwitzRadon theory [20], [21]. These codes also provide a maximum Signal-to-Noise Ratio (SNR) at receivers [22], [23]. Complex or orthogonal STBCs are more practical but it did not get the same attention by researches as the real STBCs.

\section{Technical Contribution}

The technical contribution of this paper can be summarized as follows:

3.1 The frequency-flat Rayleigh fading MIMO channel from $N_{t}$ transmit antennas to $N_{r}$ receive antennas are considered in this paper. Rayleigh fading Channel Model is a good approximation of a realistic radio channel in which there is no line of sight path between the transmitting and receiving antenna.

3.2 Using the Rayleigh fading channel, an end-to-end performance evaluation for adaptive Quadrature Phase Shift Key (QPSK) OSTBC MIMO modulation is conducted using MATLAB. The QPSK OSTBC MIMO was not fully investigated by researches as most of the previous works were investigating non-orthogonal STBC.

3.3 Implementing adaptive algorithm and test its use to control the MIMO system behavior and show how the number of transmitting and receiving antennas are adjusted adaptively to maintain the channel performance on given threshold limit of frame error rate (FER).

\section{Channel Model and OSTBC}

\subsection{Channel Model}

The channel model was analyzed by many researchers [15], [16]. In a space-time system, suppose we have $N_{t}$ transmit antennas and $N_{r}$ receive antennas. Assume that a block coded system in which $L 2^{\mathrm{M}}$ bits enter the encoder every block period. These bits are mapped to $L$ symbols, each with an $M$-array sized constellation, and transmitted over a block of $T$ time intervals. We say this is an $\left(N_{t}, N_{r}\right)$ block coded system with rate $R=L / T$. A mathematical model for any space-time block coded system is given by:

$R=S \cdot H+N$

Where:

- $R$ is a $T \times N_{r}$ matrix representing the received data.

- $S$ is a $T \times N_{t}$ matrix representing the transmitted symbols.

- $H$ is an $N_{t} \times N_{r}$ matrix representing quasi-static flat Gaussian fading.

- $N$ is a $T \times N_{r}$ matrix representing AWGN.

Only the fading and AWGN distortion are considered in the channel model shown in equation (1). For the AWGN matrix: N elements are modeled as independent circularly symmetric complex Gaussian random variables. These variables have zero mean and a variance that defines the system signal-to-noise ratio (SNR). The fading matrix, $\mathrm{H}$, is modeled in the same statistical way as AWGN with normalized unit variance [15], [16]. A quasi-static channel stays constant for a complete code block duration but may change independently from one block to another. Flat fading implies a constant power spectral density (PSD) over the frequency band used by the transmitted symbols [16]. All antennas in the system are assumed to be placed sufficiently far apart for independent fading over each channel.

\subsection{OSTBC}

Considering $M_{T} \times M_{R}$ transmission matrix with variables $s_{1}, s_{2}, \ldots$ $S n$ satisfying [2], [26], [27]:

$$
S_{M_{T}} \cdot S_{M_{T}}^{T}=c\left|s_{0}\right|^{2}+\left|s_{1}\right|^{2}+\cdots+\left|s_{n}\right|^{2} I_{M_{T}}
$$

In equation (2), $c$ is a constant and $I_{M T}$ is a $M_{T} \times M_{R}$ identity matrix. Alamouti introduced full rank diversity which was considered as the simplest STBC code with complex constellation. Alamouti's full rank diversity is the only $M_{T} \times M_{R}$ STBC code with complex constellation achieving full rate of 1 for a full diversity of 2. For 3 transmit antennas, Tarokh [19] made block codes with $1 / 2$ and $3 / 4$ code rates and full diversity $3 M_{R}$. The use of higher number of transmit antennas on generalized STBC is to achieve high capacity with full diversity, minimum decoding complexity and minimum coding delay. Examples of complex transmission matrices achieving full diversity for two, three and four transmit antennas are given as:

$$
S_{2}=\left[\begin{array}{cc}
S_{1} & S_{2} \\
-S_{2} & S_{1}
\end{array}\right]
$$




$$
\begin{aligned}
S_{3}^{C} & =\left[\begin{array}{ccc}
S_{1} & S_{2} & S_{3} \\
-S_{2} & S_{1} & -S_{4} \\
-S_{3} & S_{4} & S_{1} \\
-S_{4} & -S_{3} & S_{2} \\
S_{1}^{*} & S_{2}^{*} & S_{3}^{*} \\
-S_{2}^{*} & S_{1}^{*} & -S_{4}^{*} \\
-S_{3}^{*} & S_{4}^{*} & S_{1}^{*} \\
-S_{4}^{*} & -S_{3}^{*} & S_{2}^{*}
\end{array}\right] \\
S_{4}^{C} & =\left[\begin{array}{cccc}
S_{1} & S_{2} & S_{3} & S_{4} \\
-S_{2} & S_{1} & -S_{4} & S_{3} \\
-S_{3} & S_{4} & S_{1} & -S_{2} \\
-S_{4} & -S_{3} & S_{2} & S_{1} \\
S_{1}^{*} & S_{2}^{*} & S_{3}^{*} & S_{4}^{*} \\
-S_{2}^{*} & S_{1}^{*} & -S_{4}^{*} & -S_{3}^{*} \\
-S_{3}^{*} & S_{4}^{*} & S_{1}^{*} & S_{2}^{*} \\
-S_{4}^{*} & -S_{3}^{*} & S_{2}^{*} & S_{1}^{*}
\end{array}\right]
\end{aligned}
$$

The symbol (*) denotes the complex conjugate of the element. The matrix $S_{3}^{C}$ code transmits 4 symbols every 8time-intervals and therefore has a rate of $1 / 2$. For both schemes, flat fading channels are assumed to be constant over 8 symbol periods. For OSTBC, examples of $1 / 2$ and $3 / 4$ code rate complex transmission matrices for four transmit antennas have been proposed by Tarokh [17] which gave full diversity of $4 N_{R}$. With four transmit antennas and code rate of $1 / 2$ and $3 / 4$, complex transmission matrices have been given as:

$S_{4}^{C}\left(\frac{1}{2}\right)=\left[\begin{array}{cccc}S_{1} & S_{2} & S_{3} & S_{4} \\ -S_{2} & S_{1} & -S_{4} & S_{3} \\ -S_{3} & S_{4} & S_{1} & -S_{2} \\ -S_{4} & -S_{3} & S_{2} & S_{1} \\ S_{1}^{*} & S_{2}^{*} & S_{3}^{*} & S_{4}^{*} \\ -S_{2}^{*} & S_{1}^{*} & -S_{4}^{*} & -S_{3}^{*} \\ -S_{3}^{*} & S_{4}^{*} & S_{1}^{*} & S_{2}^{*} \\ -S_{4}^{*} & -S_{3}^{*} & S_{2}^{*} & S_{1}^{*}\end{array}\right]$

$S_{4}^{C}\left(\frac{3}{4}\right)=\left[\begin{array}{cccc}S_{1} & S_{2} & \frac{S_{3}}{\sqrt{2}} & \frac{S_{3}}{\sqrt{2}} \\ -S_{2}^{*} & S_{1}^{*} & \frac{S_{3}}{\sqrt{2}} & -\frac{S_{3}}{\sqrt{2}} \\ \frac{S_{3}^{*}}{\sqrt{2}} & \frac{S_{3}^{*}}{\sqrt{2}} & \frac{-S_{1}-S_{1}^{*}+S_{2}-S_{2}^{*}}{2} & \frac{-S_{2}-S_{2}^{*}+S_{1}-S_{1}^{*}}{2} \\ \frac{S_{3}}{\sqrt{2}} & -\frac{S_{3}}{\sqrt{2}} & \frac{S_{2}+S_{2}^{*}+S_{1}-S_{1}^{*}}{2} & -\frac{S_{1}-S_{1}^{*}+S_{2}-S_{2}^{*}}{2}\end{array}\right]$

\section{System Overview}

The proposed adaptive OSTBC MIMO communication systems over Rayleigh channel is shown in figure 1 . The system is simulated using MATLAB software.

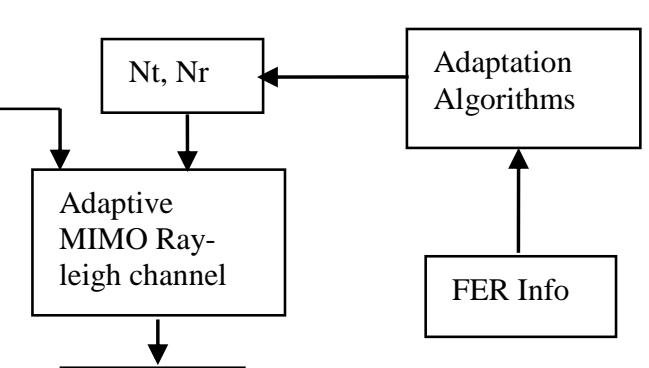

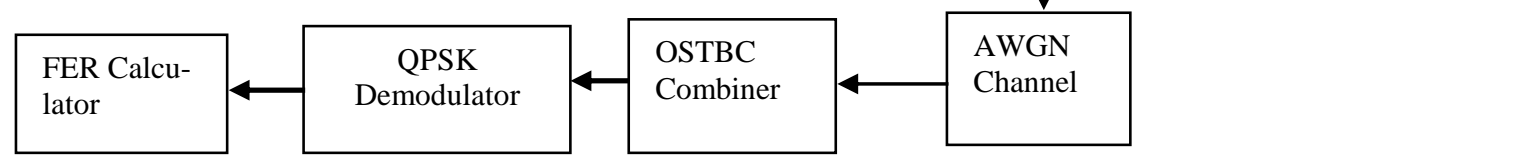

Fig. 1: System Overview

\subsection{Random Data Generator}

This block represents the information source for this simulation. It uses Bernoulli Binary Generator and generates a frame of random bits. The length of the generated frame is set to 120 bytes.

\subsection{QPSK Modulator:}

This block modulates the generated random data to a quaternary PSK (QPSK) constellation. The signal is modulated with an output size equal to half of the Bernoulli Binary Generator block output size, as every two input bits produce one modulated symbol [19].

\subsection{Orthogonal Space-Time Block Codes (OSTBC) Encoder}

This block encodes the information symbols using either the Alamouti code [ 1] for two transmit antennas or the orthogonal codes proposed by Tarokh [2] for more than two transmit antennas. In this simulation for $2 \mathrm{X} 2 \mathrm{MIMO}$, a code $\mathrm{S}_{2}$ of equation (3) is implemented while for code $S_{3}^{C}$ of equation (4) is used for
3X3 MIMO. The matrix $S_{3}^{C}$ code transmits 4 symbols every 8 time-intervals and therefore has rate $1 / 2$. For $4 X 4$ MIMO, a code $\left(S_{4}^{C}\left(\frac{1}{2}\right)\right)$ shown in equation (6) proposed by Tarokh [17] is implemented. This code gives full diversity of $4 N_{R}$.

\subsection{Adaptive MIMO Raleigh Channel}

This block simulates the frequency-flat Rayleigh fading MIMO channel. The block is configured as a spatially independent 4x4 MIMO channel with transmit and receive antenna selection. The parameters for this model is set so that the MIMO channel behaves like a quasi-static fading channel.

\subsection{AWGN}

This block adds white Gaussian noise at the receiver side.

\subsection{OSTBC Combiner}

This block combines the received signal with the channel state information (CSI) to output the estimates of the modulated sym- 
bols. In this simulation, the CSI is assumed to be known at the receiver side.

\subsection{QPSK Demodulator}

This block demodulates the signal recovered from OSTBC Combiner.

\subsection{FER Calculator}

The FER Calculator calculates the system FER by comparing the decoded bits with the original source bits per frame to detect errors and dynamically updates the FER along the number of error frames observed, and the number of frames processed. The model also controls the duration of the simulation. The simulation stops when the target number of error frames is reached (in case of noisy environment) or when all number of frames are transmitted whichever comes first.

\section{Simulation Results}

\subsection{Simulation Parameters:}

The simulation parameters are shown in table 1 .

Table 1: Simulation parameters

\begin{tabular}{|c|c|c|}
\hline \multirow[t]{6}{*}{ General Parameters } & Parameters & Value \\
\hline & Frame length & 120 bytes \\
\hline & SNR & $0 ; 5 ; 10 ; 15 ; 20 \mathrm{~dB}$ \\
\hline & Target FER & $2.0 \mathrm{e}-1$ \\
\hline & Max Error count & 500 frames \\
\hline & Max frame count & $1 e+04$ \\
\hline \multirow{4}{*}{$\begin{array}{ll}\text { Bernolli } & \text { Binary } \\
\text { Generator } & \end{array}$} & Probability of zero & 0.5 \\
\hline & Initial seed & 61 \\
\hline & Sample time & $1 e-6$ \\
\hline & Output data type & Binary \\
\hline \multirow[t]{3}{*}{ QPSK modulator } & Input type & Bit \\
\hline & $\begin{array}{l}\text { Constellation order- } \\
\text { ing }\end{array}$ & Gray \\
\hline & Phase offset (rad) & 0 \\
\hline \multirow{9}{*}{$\begin{array}{ll}\text { MIMO } & \text { Fading } \\
\text { Channel } & \end{array}$} & Frequency selectivity & Multipath parameter \\
\hline & Sample rate $(\mathrm{Hz})$ & $1 \mathrm{e} 6 / 3$ \\
\hline & $\begin{array}{l}\text { Discrete path delays } \\
\text { (s) }\end{array}$ & 0 \\
\hline & $\begin{array}{l}\text { Average path gains } \\
\text { (dB) }\end{array}$ & $0 \mathrm{~dB}$ \\
\hline & Fading distribution & Raleigh; Rician \\
\hline & $\begin{array}{l}\text { Maximum Doppler } \\
\text { shift }(\mathrm{Hz})\end{array}$ & 100 \\
\hline & Doppler spectrum & Doppler ('Jakes') \\
\hline & Spatial correlation: & $\begin{array}{l}\text { None; Separate TX Rx; } \\
\text { Combined }\end{array}$ \\
\hline & $\begin{array}{l}\text { Number of transmit } \\
\text { antennas }\end{array}$ & $1,2,3,4$ \\
\hline
\end{tabular}

\begin{tabular}{|l|l|l|}
\hline \multirow{2}{*}{ AWGN } & $\begin{array}{l}\text { Number of receive } \\
\text { antennas }\end{array}$ & $1,2,3,4$ \\
\hline \multirow{2}{*}{ QPSK Demodulator } & Noise method & Variance \\
\cline { 2 - 3 } & Initial seeds & 67 \\
\cline { 2 - 3 } & $\begin{array}{l}\text { Constellation order- } \\
\text { ing }\end{array}$ & Gray \\
\hline
\end{tabular}

\subsection{Results and Discussion}

\subsubsection{Mimo System Performance}

The FER of the adaptive MIMO system is calculated for different SNR and different number of transmitted and received antennas to test the improvements that can be obtained when using different MIMO configurations. As a general simulation note, for the assumed frequency-flat Rayleigh fading MIMO channel model, it is shown that a higher number of transmitting and receiving antenna elements gives higher performance. Figure (2) shows the calculated FER for different SNR and different number of transmitted and received antennas. It is shown from figure (2) that for $1 \mathrm{X} 1$ antenna configuration, the lowest performance was attained, and the FER improved after $\mathrm{SNR}=15 \mathrm{~dB}$ while the FER improved after $\mathrm{SNR}=10 \mathrm{~dB}$ for $2 \mathrm{X} 2$ antenna configuration. For $3 \mathrm{X} 3$ it is improved after $\mathrm{SNR}=8 \mathrm{~dB}$ and for $4 \mathrm{X} 4 \mathrm{MIMO}$ it is improved after $\mathrm{SNR}=7 \mathrm{~dB}$.

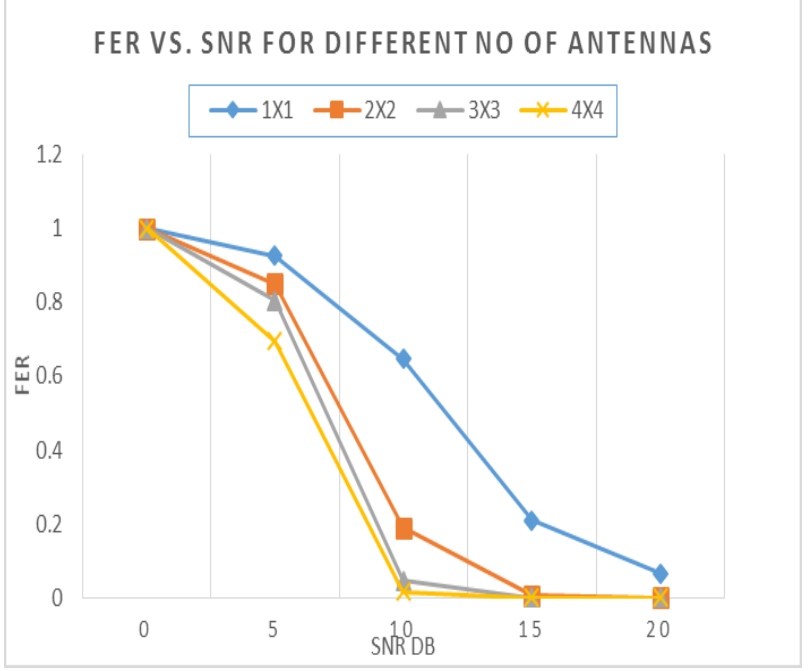

Fig. 2: FER Vs. SNR for different Antenna configuration

\subsubsection{Constellation Diagram}

The QPSK is the most robust modulation. It can be represented by a constellation. The received constellation is generated for QPSK OSTBC MIMO system with 4X4 antennas for different SNR. The results are shown in figure (3). It is shown from Figure (3) that as the SNR improves and the received constellation symbols become better as the constellation remain constant over big number of symbols.

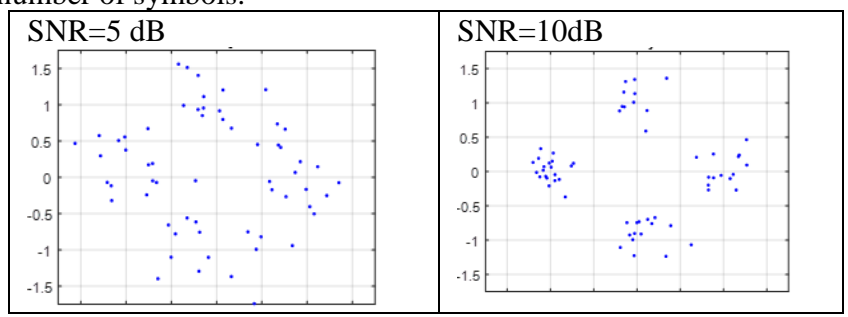




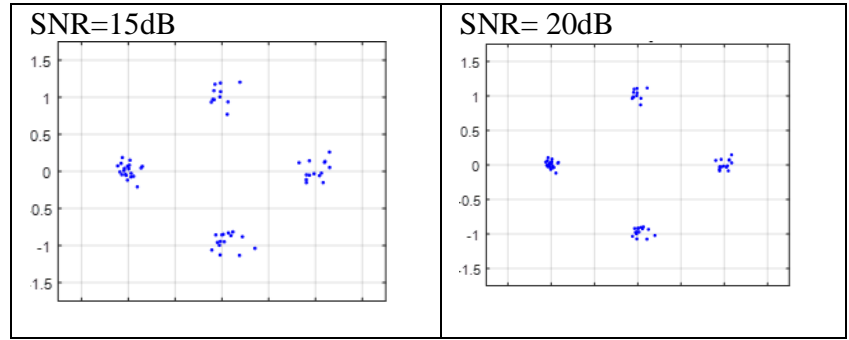

Fig. 3: Constellation diagram for Different SNR using MIMO with 4X4 antennas

\subsubsection{Testing the Adaptation Algorithm}

To test the adaptation algorithm, a target FER is set to 2.0e-1. Four simulation rounds are done using $\mathrm{SNR}=5,10,15$, and $20 \mathrm{db}$. For each SNR round, the number of MIMO transmitted and received antennas adjusted by the proposed system is monitored. The number of MIMO antennas is set automatically by the adaptation algorithm to maintain system FER below or equal to the target FER (2.0e-1). The summary of percentage usage of each MIMO antenna combinations for each case of SNR are shown in table (2).

Table 2: Summary of the usage of transmitted and received antenna for different SNR

\begin{tabular}{|l|l|l|l|l|}
\hline SNR & $\begin{array}{l}1 \mathrm{X} 1 \text { antenna } \\
\text { Usage }\end{array}$ & $\begin{array}{l}2 \mathrm{X} 2 \text { antenna } \\
\text { usage }\end{array}$ & $\begin{array}{l}3 \mathrm{X} 3 \text { antenna } \\
\text { Usage }\end{array}$ & $\begin{array}{l}4 \mathrm{X} 4 \text { antenna } \\
\text { usage }\end{array}$ \\
\hline $5 \mathrm{~dB}$ & $0.6 \%$ & $0.6 \%$ & $0.6 \%$ & $98.2 \%$ \\
\hline $10 \mathrm{~dB}$ & $47.9 \%$ & $14.7 \%$ & $8.8 \%$ & $28.6 \%$ \\
\hline $15 \mathrm{~dB}$ & $76.1 \%$ & $9.3 \%$ & $8.1 \%$ & $6.5 \%$ \\
\hline $20 \mathrm{~dB}$ & $100 \%$ & $0.0 \%$ & $0.0 \%$ & $0.0 \%$ \\
\hline
\end{tabular}

It is shown from table (2) that for low SNR value the system depends heavily on $4 \mathrm{X} 4$ antenna configuration and as SNR becomes better the system switch between many combinations of antennas as the case for $\mathrm{SNR}=10$ and $15 \mathrm{~dB}$. When $\mathrm{SNR}$ reaches $20 \mathrm{~dB}$ the system relies completely on $1 \mathrm{X} 1$ antenna configuration for transmitting and receiving.

Figure (4), (5), and (6) are drawn for $S N R=10 d B$. Figure (4) shows the FER vs. time while figure (5) and (6) show the number of MIMO transmitted and received antennas selected by the adaptation algorithm respectively. It is shown that the adaptation algorithm quickly succeeded in adjusting FER shown in figure (4) on its setup limit by dynamically adjusting the usage of transmitted and received MIMO antennas, shown in figure (5) and (6), to overcome the impairment caused by the faded Gaussian channel condition for the given SNR (10dB).

\section{Conclusion}

In this paper, end-to-end adaptive Orthogonal Space-Time Block Codes (OSTBC) Multiple Input Multiple Output (MIMO) communication systems was simulated over frequency selective Rayleigh fading channel using Frame Error Rate as a performance measures. For different SNR, the system FER and constellation symbol diagram was calculated. For a given SNR, the calculated FER and Constellation symbol diagram is shown to improve as more antennas are used. It is also shown that the adaptation algorithm quickly succeeded in adjusting FER on its setup limit by dynamically adjusting the usage of transmitted and received MIMO antenna to overcome the impairment caused by the faded Gaussian channel condition for a given SNR. As a future work extending our 4X4 MIMO model to 8X8 MIMO as well as more modulation schemes like 4-QAM and 16-QAM could tested.

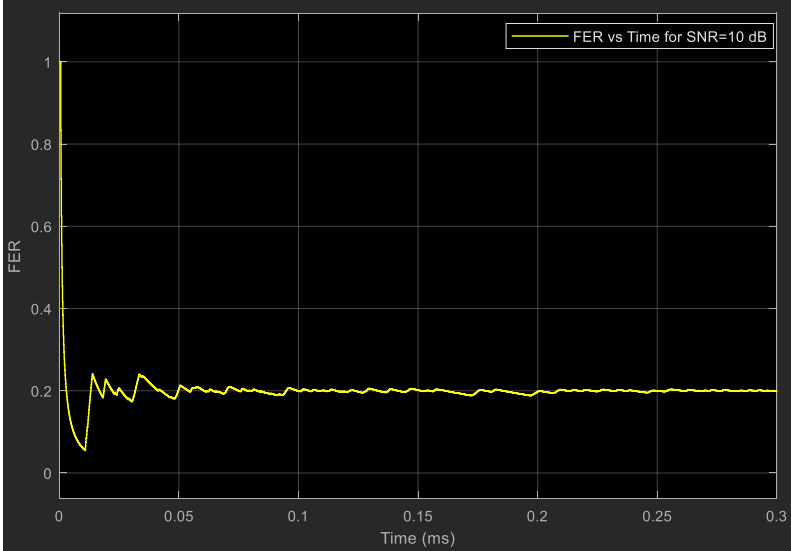

Fig. 4: FER vs. Time for $\mathrm{SNR}=10 \mathrm{~dB}$

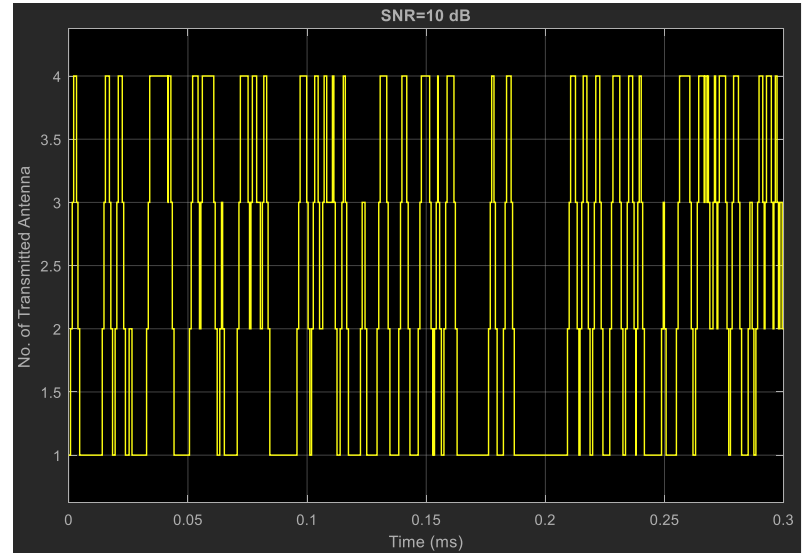

Fig. 5: Number of transmitted antenna Vs. Time for $S N R=10 d B$

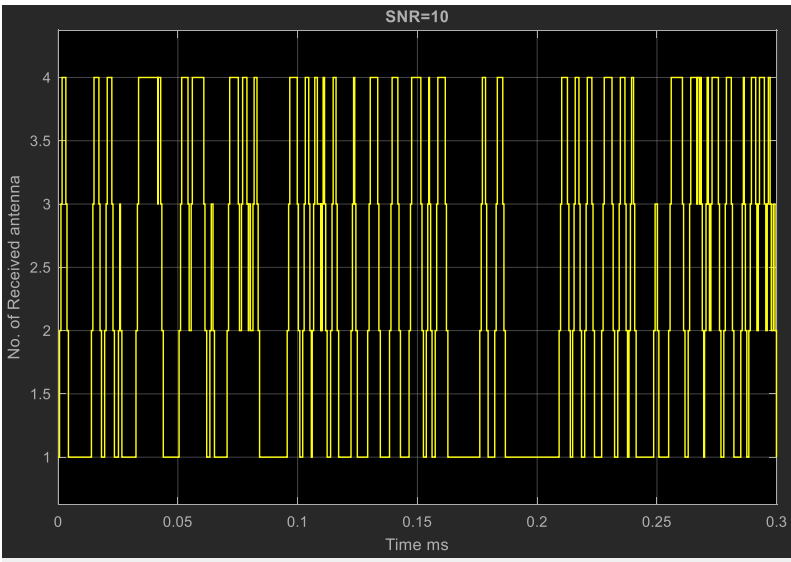

Fig. 6: Number of received antenna Vs. Time for $S N R=10 d B$

\section{References}

[1] S. M. Alamouti, "A simple transmit diversity technique for wireless communications," IEEE Journal on Selected Areas in Communications, Vol. 16, No.8, (1998), pp: 1451-1458. DOI: $10.1109 / 49.730453$.

[2] V. Tarokh, H. Jafarkhami, and A. R. Calderbank, 1999, "Spacetime block codes from orthogonal designs," IEEE Transactions on Information Theory, Vol. 45, No.5, (1999), pp: 1456-1467. DOI: $10.1109 / 18.771146$

[3] Goldsmith, A., Wireless Communications, first edition, Cambridge University Press, Cambridge, England, 2005.

[4] Foschini, G. J. and Gans, M. J., "On limits of wireless communications in a fading environment when using multiple antennas," Wireless Communications, vol. 6, No.3, (1998), pp: 311-335, DOI: https://doi.org/10.1023/A:1008889222784

[5] Chen-nee C., David N. C., Joseph M. K. and Reinaldo A. V., "Capacity Scaling in MIMO Wireless Systems under Correlated Fad- 
ing" IEEE Transactions on Information Theory, Vol. 48, No. 3, (2002), pp: 657-649, DOI: 10.1109/18.985982.

[6] Tobias W., Alexandros S., and Michael M., "Imperfect ChannelState Information in MIMO Transmission" IEEE transact ions on communication, Vol. 54, No.3, (2006), pp 543-552.

[7] David T. and Pramod V., "Fundamentals of Wireless Communications", Cambridge University Press, United Kingdom, (2005), pp: $49-111,166-217$ and $290-483$

[8] Okh, V., Naguib, A., Seshadri, N., and Calderbank, A.R., "Spacetime codes for high data rate wireless communication: Performance criteria in the presence of channel estimation errors, mobility, and multiple paths," Communications, IEEE Transact ions, Vol. 47, No. 2, (1999), pp: 199-207.

[9] Tarokh, V., Jafarkhani, H. and Calderbank, A. R., "Space-time block coding for wireless communications: performance result s", IEEE Journal of selected areas in Communications, Vol. 17, No. 3 (1999), pp: 451-460.

[10] Ganesan, G and Stoica, P., "Differential detection based on spacetime block codes," Wireless Personal Communications, vol. 21, No. 2, (2002), PP: 163-180.

[11] Golden, G. D, Foschini, C. J, Valenzuela, R. A and Wolniansky, P. $\mathrm{W}$, "Detection algorithm and initial laboratory results using VBLAST space-time communication architecture," IEEE Letters, Vol. 35, No.1, (1999), pp: 14-16.

[12] Lai T. X., T ran T.A. and Sessay A.B.," Performance Analysis of Space Time Block Coded Systems over Frequency Selective Rayleigh Fading Channels, " EEE Wireless Communications and Networking Conference, Vol.3, (2006), pp: 1480-1485, DOI:10.1109/WCNC.2006.1696506.

[13] Cortes-Pena L, "MIMO Space-Time Block Coding (STBC): Simulation and Results" ECE6604 Personal and Mobile Communications, (2009), pp: 1-8.

[14] Hyoungju Ji, Younsun Kim, and Juho Lee," Overview of FullDimension MIMO in LTE-Advanced Pro", IEEE Communications Magazine, Vol 55, no. 2, (2017), pp: 176-184.

[15] W. C. Jakes, Ed., Microwave Mobile Communications, New York: Wiley, (1974).

[16] G. L. Stuber, Principles of Mobile Communication, Norwell, MA: Kluwer Academic Publishers, 2nd edition, (2001).
[17] V. Tarokh, N. Seshadri and A. R. Calderbank, "Space-time codes for high data rate wireless communication: performance criterion and code construction," IEEE Trans. Inform. Theory, vol. 44, No. 2, (1998), pp: 744-765.

[18] Foschini, G. J. "Layered space-time architecture for wireless communication in a fading environment when using multi-element antennas. Bell Labs Technical Journal, vol. 1, No. 2, (1998), pp: 41-59.

[19] Hochwald, B. M. and Marzetta, T. L., "Unitary space-time modulation for multiple-antenna communications in Rayleigh flat fading”, IEEE Trans. Inform. Theory, 46(2), 2000, 543-564.

[20] Ganesan, G. and Stoica, P., "Space-time diversity using orthogonal and amicable orthogonal designs", Proc. IEEE Int. Conf Acoust., Speech, and Signal Processing ICASSP '00, Vol. 5, (2000), pp:2561-2564.

[21] Geramita, A. V. and Seberry, J., "Orthogonal designs: quadratic forms and Hadamard matrices", Lecture notes in pure and applied mathematics, Marcel Dekker, New York and Basel. Godara, vol. 23, No.1, (1997). pp: 116-117.

[22] Ganesan, G. and Stoica, P.," Space-time block codes: a maximum SNR approach", IEEE Trans. Inform. Theory, Vol. 47, No. 4, (2001), pp: $1650-1656$.

[23] R.O. Abolade, Z. K. Adeyemo," Adaptive Modulation Performance In Mimo- OSTBC Over Rayleigh Fading Channel", International Journal of Engineering \& Computer Science IJECSIJENS, Vol. 12, No. 6, (2012), pp: 21-25.

[24] A. Molisch, Wireless Communications, Wiley-IEEE Press, (2005).

[25] D. Gesbert, M. Shafi, D.-S. Shiu, P. J. Smith, and A. Naguib, "From theory to practice: an overview of MIMO space-time coded wireless systems," IEEE Journal on Selected Areas in Communications, Vol. 21, No. 3, (2003), pp:281-302.

[26] B. Vucetic and J. Yuan, Space-Time Coding, JohnWiley \& Sons, New York, NY, USA, (2003).

[27] Ravi Kumar, Rajiv Saxena, "Analysis of Adaptive Semiblind Channel Estimation Scheme with Missed Data Channel Estimation Schemes in MIMO Antenna system" International Journal of Computer Application, Vol.59, No.4, 2012, pp:33-38, DOI: 10.5120/9537-3973 